\title{
On Changing Patterns of Morphological Instability in Linear-Low-Density Polyethylenes with Different Methods of Synthesis
}

\author{
Ian Lee Hosier and David Clifford BASSETT \\ J. J. Thomson Physical Laboratory, University of Reading, \\ Reading RG6 6AF, U.K.
}

(Received January 5, 1999)

\begin{abstract}
In contrast to linear polymers, linear-low-density polyethylenes (with more than $\sim 10$ branches per $1000 \mathrm{C}$ atoms) crystallizing from linear nuclei to form row structures do not crystallize uniformly. Instead they pass through a series of morphological changes, accompanied by a continuously declining growth rate, because of the accumulation of more branched molecular sequences segregated at the growth front and the consequent progressive decrease in isothermal supercooling. Different concentrations of segregants, reflecting the different interfacial geometries, give spherulites - with their more open construction - a faster growth rate than rows and render the rows more susceptible to morphological instability. Nevertheless, cellulation does occur in spherulites of a metallocene polymer with 28 ethyl branches per $1000 \mathrm{C}$ atoms. Systematic differences in morphology and relative growth rate between metallocene and Ziegler Natta-catalyzed polymers are attributed to less mobile segregants for the former. There is no evidence for segregation in row structures of commercial linear polyethylenes except for tiny proportions of possibly shorter molecules in pockets of subsidiary lamellae.

KEY WORDS Morphology/Linear-Low-Density Polyethylenes/Row Structures/Electron Microscopy / Metallocene /
\end{abstract}

Recent work ${ }^{1}$ has shown how phenomena of morphological instability can appear during the crystallization of linear-low-density polyethylenes on linear nuclei. Experimentally this is a new phenomenon although it is linked to the theoretical concept of cellulation proposed long $\mathrm{ago}^{2}$ as a possible cause of spherulitic growth. It is a consequence of segregation of certain molecular species at the crystal/melt interface causing the growth rate to decline and the interface to become potentially unstable. Its presence in linear-low-density polyethylenes is a consequence of the rejection of more-branched molecular sequences which lower the local equilibrium melting temperature and with it the isothermal supercooling and the growth rate. Conversely its absence in the linear polymer is because any segregation of shorter molecules leaves the growth rate little affected.

The study of morphological instability is better carried out using linear nuclei, to form row structures, than for spherulites-which effectively grow from points-because the consequent epitaxial growth is constrained to place lamellae in close-packed parallel array thereby maximizing the concentration of segregants at the growth interface as well as making for easier observation and measurement. We report in this paper the first comparative study of morphological instability and cellulation in row structures of a series of linear-lowdensity polyethylenes differing in branch content and method of synthesis. There are two principal findings. One, which has been followed up elsewhere ${ }^{3,4}$ is that cellulation occurs in spherulites as well as in rows for polymer of high branch content. The second is that the behavior displays different characteristics according to whether it was synthesized by Ziegler-Natta means or using a metallocene catalyst. Segregants appear to be more mobile in the former case, possibly because they consist of entire molecules rather than segments. It is possible that morphological instability and cellulation may offer a useful means of differentiating between such materials.

\section{EXPERIMENTAL}

The eight polymers employed in this investigation are listed in Table I. LL0209 and both Rigidex polymers are products of BP Chemicals; Sclair 2907 is from du Pont (Canada) while polymers A, B, C, and Z were kindly supplied by Dr P Jääskeläinen of Borealis Oy, Finland. They represent a wide cross section through commonly available polyethylenes, both high and low density and include both different synthesis methods and branching levels. Row structures were prepared in them and examined following previous techniques ${ }^{1}$ but with minor refinements.

First a flat disc was prepared from a single pellet of the chosen polymer by melt pressing, between clean microscope slide and cover slip, on a Kofler WME hot-bench at $160^{\circ} \mathrm{C}$ for $5 \mathrm{~min}$ turning regularly to ensure a constant temperature. This procedure allowed the stresses associated with pressing to relax and gave a disc of about $2 \mathrm{~mm}$ in thickness which, after this time, was quenched into an ice/water mixture. The top cover slip was subsequently peeled away allowing bundles of Tekmilon* advanced polyethylene fibers, nominally 40 microns in diameter, to be placed over the surface of the pressed disc and secured by being attached, away from the sample, to a supporting glass slide using a quick-drying contact cement. With the fibers in place, the entire composite was then re-pressed at $130^{\circ} \mathrm{C}$ which resulted in the Tekmilon fibers being located some $60 \mu \mathrm{m}$ below the surface (particular care being taken at this

* These fibers, manufactured by Mitsui Petrochemical by a process of melt-kneading, have a molecular weight of $7 \times 10^{5}$ Daltons and a tensile modulus of $62 \mathrm{GPa}$. 
stage to ensure that the fibers did not melt). The sample was held at $130^{\circ} \mathrm{C}$ for a further 3 min then immediately transferred to an adjacent Mettler FP5 hot-stage maintained at the required crystallization temperature. These were chosen to give approximately the same size of row for all samples. After $30 \mathrm{~min}$ the specimen was quenched immediately into ice/water mixture, later removed, rinsed with distilled water and dried under vacuum for $1 \mathrm{~h}$.

Growth from the embedded fibers was viewed following permanganic etching. This was for $4 \mathrm{~h}$ in a $1 \%$ $(\mathrm{w} / \mathrm{v})$ potassium permanganate solution in a mixed solvent composed of 1 part of dry phosphoric acid to 2 parts of sulphuric acid. Samples were recovered in a $3 \%$ solution of hydrogen peroxide, rinsed in distilled water and acetone and then vacuum dried. The etching removed $30-40$ microns of the top layer of the material exposing a cross-section close to the fiber axis. This etched surface was replicated using a standard two-stage technique producing shadowed carbon replicas for examination in the transmission electron microscope.

\section{RESULTS AND DISCUSSION}

Row structures ${ }^{5}$ are a well-known feature of polymeric systems. They are related to, but should be differentiated from, transcrystalline layers. ${ }^{6}$ Row structures form by epitaxial growth from linear nuclei in which lamellae share the chain axis direction of the nucleus and grow out transversely. The fastest growth direction (b in polyethylene, $\mathbf{a}^{*}$ in $\alpha$-polypropylene) becomes radial within a cylindrical envelope centered on the nucleus. Transcrystalline layers, on the other hand, consist of lamellae growing out from a nucleating surface. The fastest growth direction becomes normal to this surface but lamellae can adopt any orientation around it so that, unlike row structures, there is no common chain axis direction.

For isothermal growth, row structures and transcrystalline layers of linear polyethylene or isotactic polypropylene ${ }^{7}$ increase their width linearly with time, showing the same isothermal growth rates as spherulites of the same materials. This is a consequence of a uniform outward growth of lamellae. The discovery ${ }^{1}$ that this is not the case for a certain linear-low-density polyethylene, for which there is a sequence of morphological changes accompanying a continuous decline in growth rate, has revealed a new phenomenon which is explored further here. Both morphological changes and declining kinetics are consistent with a progressive decrease in isothermal supercooling at the growth front. It is observed that the texture coarsens with adjacent lamellae moving farther apart on average, allowing deviations from the radial growth direction to occur together with a modest increase of thickness. These changes have since been quantified by Abo el Maaty ${ }^{8}$ who has shown, by crystallizing at higher temperatures than those employed here or previously, ${ }^{1}$ that they eventually stabilize, with the declining growth rate, and asymptotically approach a steady state. In this condition the rate of addition of segregants from crystallization is equal to the rate of loss by diffusion but the growth interface resides within the segregant-rich region.

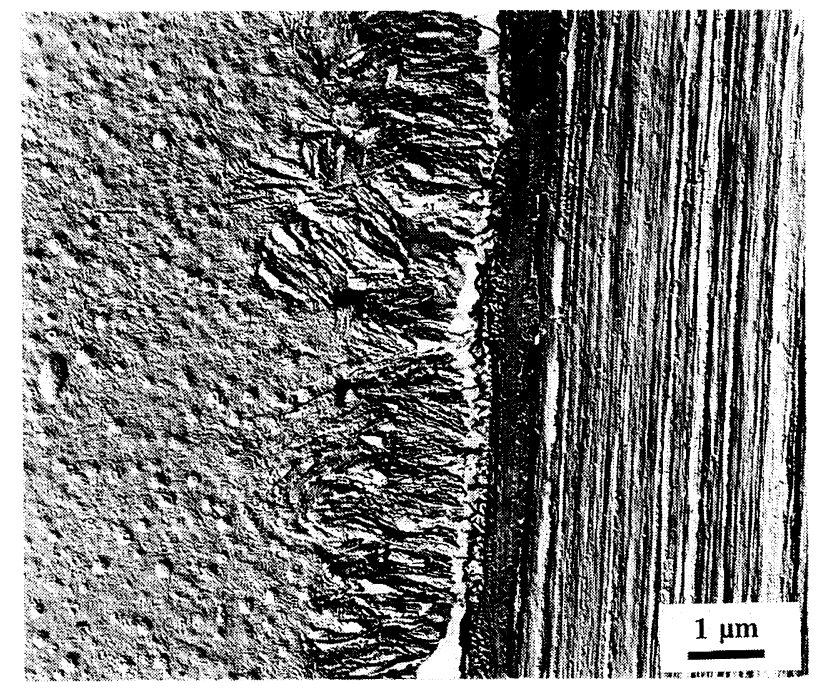

Figure 1. Details of growth patterns in LL0209 showing how the initial uniformity with lamellae growing normal to the nucleating fiber becomes unstable with the development of spherulitic protrusions.

While these changes are predictable and in accord with expectation given the nature of the segregated molecules, as is the fact that spherulites elsewhere in the sample grow faster than rows, ${ }^{1}$ because of their more open interfacial structure-which reduces segregant concentrations-there is also an entirely unexpected phenomenon. This is the formation at the instantaneous interface, when growth has slowed substantially, of wellseparated nuclei with a different crystallographic orientation which are thereby no longer constrained to grow as part of the row but are able to develop in an essentially spherulitic manner. As this is a faster mode, for the reason cited above, such overgrowths proceed to develop as faster-growing protrusions ahead of the remainder of the row as shown in Figure 1. Such instability was only observed ${ }^{1}$ for rows, never for spherulites in the same samples because, it was suggested, for them the segregant concentration did not reach a critical level. This consideration prompted the present study across several different polyethylenes seeking both to confirm and to elaborate the circumstances in which these new phenomena occur.

The material of Figure 1 is the same as that of the previous study; it has the second highest branch content of the linear-low-density polyethylenes considered. Our observations agree with the previous report ${ }^{1}$ in that transverse growth from the fiber begins in close-packed array, then coarsens with adjacent lamellae becoming further apart on average and somewhat thicker.

The two linear-low-density polyethylenes with least branch content available to us were polymers $\mathrm{A}$ and $\mathrm{B}$. They have the same average branch content but were prepared differently; the former is a metallocene material while the latter was prepared by Ziegler-Natta catalysis. Earlier work ${ }^{9}$ on polymers $\mathrm{A}, \mathrm{B}$, and $\mathrm{C}$ showed that only the last two contained a proportion of inter-branch sequences sufficiently long to enter the high pressure hexagonal phase. It follows that $\mathrm{B}$ (and also $\mathrm{C}$ ) contains more short inter-branch separations than $\mathrm{A}-$ which will be segregated because of the exclusion of branches from the lattice. The greater chemical regularity of the 


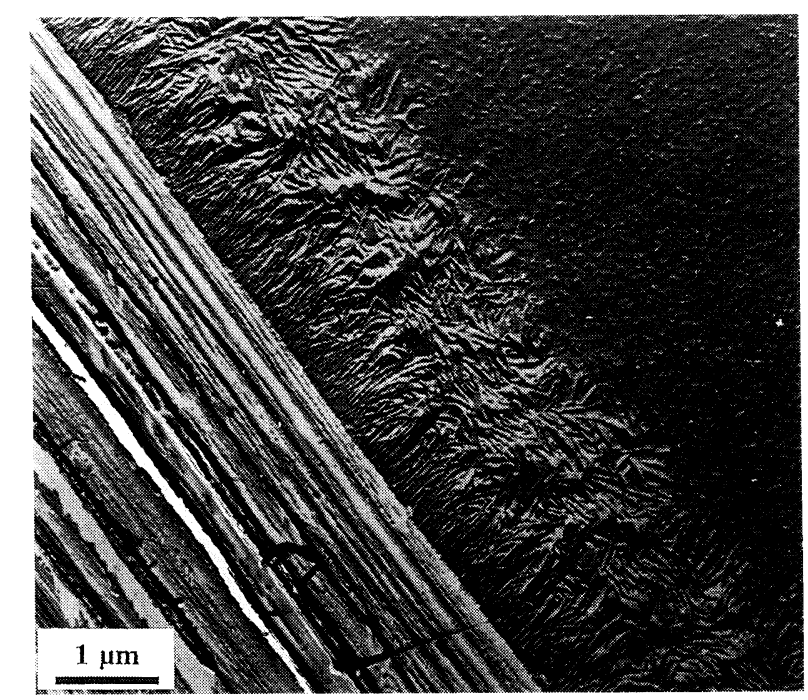

Figure 2. Details of lamellar growth in a row of the metallocene polymer A. Note that lamellae become thicker and further apart on average at increasing radial distance but the interface remains rather uniform.

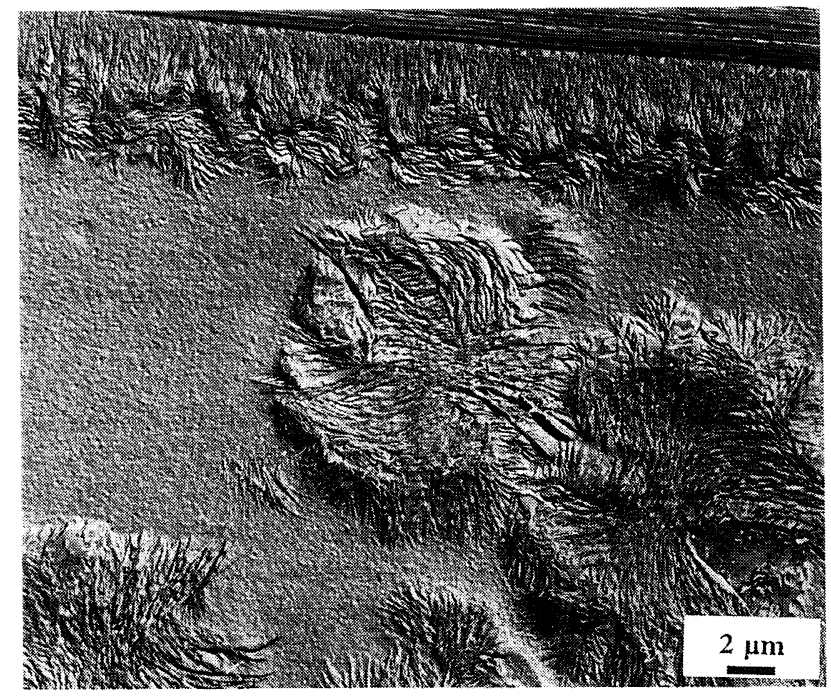

Figure 3. In the Ziegler-Natta polymer B, with the same average branch content as polymer $\mathrm{A}$, the interface is highly corrugated and spherulitic protrusions have begun to develop. The radii of the adjacent spherulites are larger than that of the row showing that they have grown the faster-a consequence of their more open structure.

metallocene material and differences between the two Ziegler-Natta polymers are reflected in the morphology of their respective rows.

For polymer A initially growth of thin close-packed lamellae proceeds radially outwards from the fiber. Figure 2 shows the development of coarsening with radial distance so that by the outer interface lamellae are well-separated and thicker than those adjacent to the fiber. In addition growth has become banded as is the case for growth at higher supercooling. However the trace of the envelope of the interface is still rather linear.

Polymer B, with the same average branch content shows a similar sequence to polymer A but with subtle and significant differences (Figure 3). The initial growth is again of close-packed planar lamellae following uni-

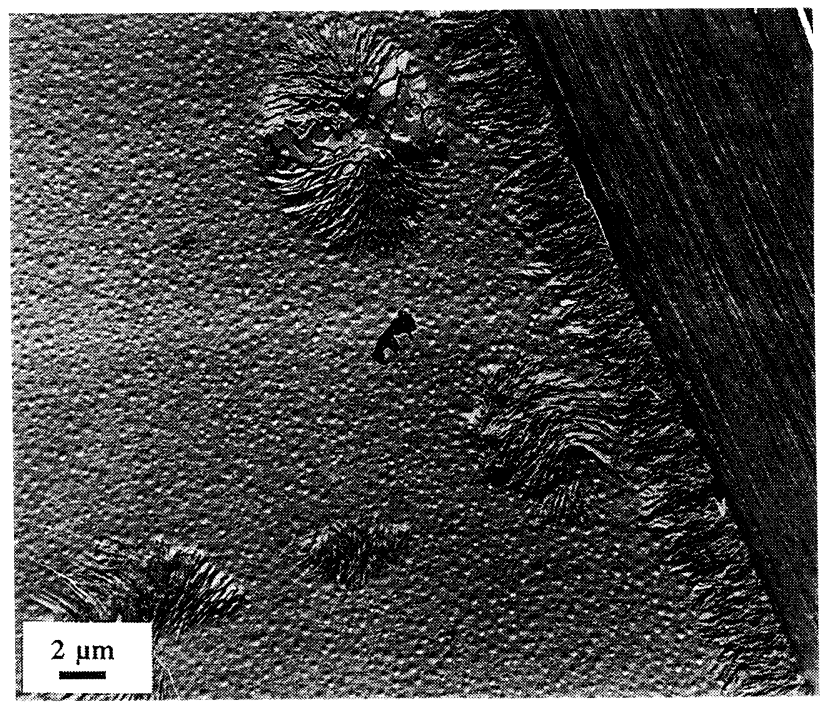

(a)

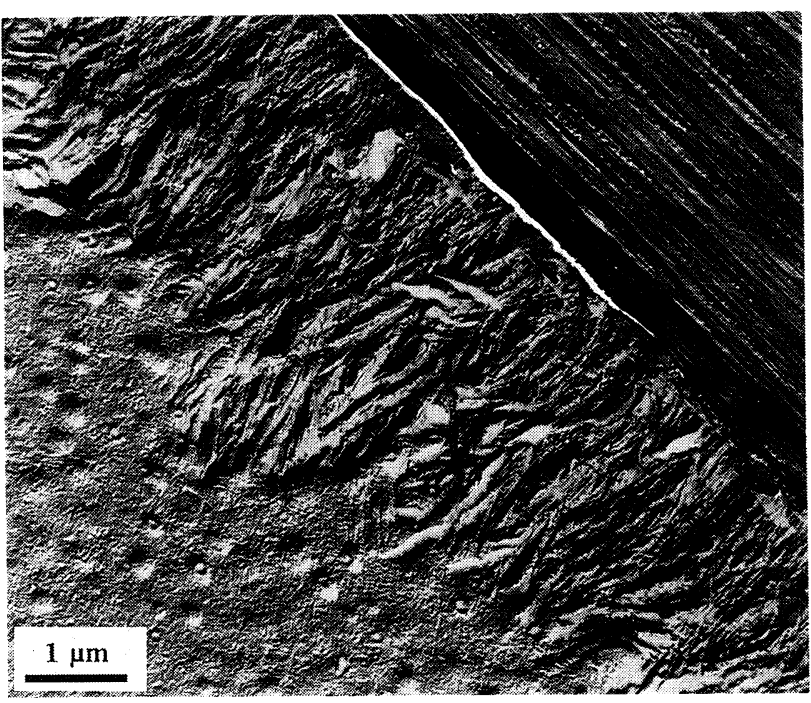

(b)

Figure 4. (a) Overview of epitaxial growth in polymer $\mathrm{C}$ showing the jagged interface. (b) Details of growth in polymer $\mathrm{C}$ showing a structure with lamellar protrusions rather similar to that of LL0209.

form nucleation along the fiber. However, the subsequent changes leave the trace of the final interface much less linear than in A with several protrusions. Notice also that the radii of the two large spherulites in Figure 3-in which the open character of the diverging dominant lamellae is clearly evident-are longer than that of the row indicating a correspondingly faster rate of growth. This contrasts with polymer A where the difference in radius was much less marked. The trend continues with polymer $\mathrm{C}$ which has the same density as polymer $\mathrm{A}$ but a higher branch content and a reduced proportion of longer inter-branch sequences able to enter the high pressure hexagonal phase. In this case the row morphology moves further towards the behavior of the LL0209 material. Figure 4a contrasts a row with a nearby faster-growing spherulite while very noticeable protrusions are present in Figure $4 b$.

We may conclude from the comparison of polymers $\mathrm{A}$ and $\mathrm{B}$ that it is the nature of the branching distribution rather than its average magnitude which causes the 
difference in behavior. It has previously been shown ${ }^{9}$ that $\mathrm{B}$ contains a greater proportion of long inter-branch sequences than $\mathrm{A}$ which, because the average branch content is the same, implies that polymer B must contain more short inter-branch sequence lengths. It is they which are more prone to rejection from the crystal surface because butyl branches are known to be excluded from the crystal lattice. ${ }^{10}$ By the same argument the demonstration that there are fewer long inter-branch sequences implies that there are yet more of such species in the more highly branched polymer $\mathrm{C}$ which, accordingly, shows a greater tendency to instability. The implication of the greater disparity in growth rate between rows and spherulites in polymer B than for A is that the segregants appear to be less mobile in polymer A.

This is in accord with the belief that branching is more regularly distributed in metallocene materials than in their Ziegler-Natta counterparts for which the shorter molecules tend to be the more branched. ${ }^{11}$ It may be that in the former it is segments rather than whole molecules which are rejected so that the local concentration of segregants is less reduced by diffusion into the open construction of spherulites than for the latter where molecules are free to move as a whole. This inference is also consistent with the explanation offered previously for the formation of the faster-growing protrusions. It was suggested ${ }^{1}$ that nucleation occurred when fluctuations allowed segregant concentration to reach a sufficient value. More mobile segregants in Ziegler-Natta polymers would allow greater fluctuation in their concentration thereby promoting the observed effect. Still further support comes from the subsequent observation $^{8}$ that this type of protrusion does not develop in LL0209 at high temperatures when the higher diffusion rate and slower growth rate combine to reduce the local segregant concentration.

The most-branched linear-low-density polyethylene available to us was polymer $\mathrm{Z}$, a metallocene material. In Figure 5 a length of row structure extends more or less uniformly out to the black impurity particle with a spherulite beyond it. While it is less easy to see lamellar detail than hitherto the difficulties of achieving this resolution in highly-branched materials should not be underestimated. Figure 5 shows, nevertheless, two important features. The first is that as for the other metallocene polymer, $\mathrm{A}$, the radius of the spherulite is again close to that of the row, reinforcing the finding of a smaller difference in the two growth rates, and by implication reduced segregant mobility, for metallocene polymers. Of exceptional interest is that the spherulite itself has developed a wavy profile. This is cellulation identified, we believe, for the first time in an undoped polymer. It differs from the previous case in that the protrusions in rows are faster-growing than the row whereas here the growth rate of the envelope slows continuously towards its asymptotic limit. Abo el Maaty has since shown ${ }^{12}$ how this is translated into the growth of the individual cellulated units with slower growing 'fibrils' being overtaken by others, less impeded, from the rear. Subsequent research on this polymer and others showing similar behavior has since been published elsewhere. $^{3,4}$

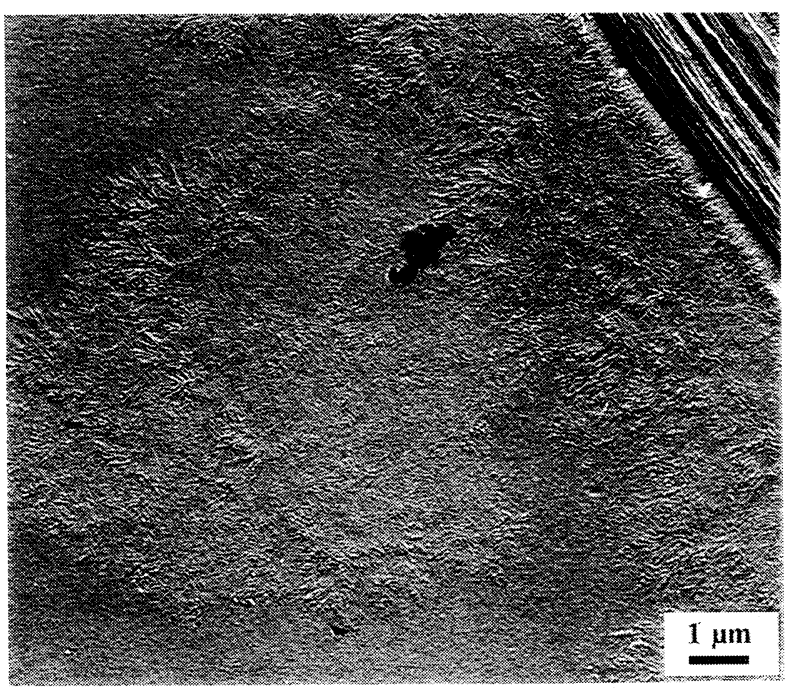

Figure 5. Lamellar detail of a row in polymer Z, extending to the black particle and a spherulite beyond whose wavy perimeter shows that it has cellulated.

Table I.

\begin{tabular}{|c|c|c|c|c|c|c|}
\hline Polymer & Catalyst & $T_{\mathrm{m}}^{\mathrm{a}} /{ }^{\circ} \mathrm{C}$ & $T_{\mathrm{c}}^{\mathrm{b}} /{ }^{\circ} \mathrm{C}$ & $\begin{array}{l}\text { Branches } \\
\text { per } 1000 \\
\text { C atoms }\end{array}$ & $M_{w}$ & $M_{n}$ \\
\hline LL0209 & $\mathrm{ZN}^{\mathrm{c}}$ & 125 & 121 & 21 butyl & 126000 & 30300 \\
\hline Sclair 2907 & $\mathrm{ZN}^{\mathrm{c}}$ & 131.5 & 127.5 & 2 & 104000 & 11350 \\
\hline $\begin{array}{l}\text { Rigidex } \\
140 / 60\end{array}$ & $\mathrm{ZN}^{\mathrm{c}}$ & 131.5 & 127.5 & 3 & 53000 & 16000 \\
\hline Rigidex 9 & $Z^{c}$ & 131.5 & 127.5 & 2 & 170000 & 11300 \\
\hline A & $\mathbf{M}^{\mathrm{d}}$ & 117 & 113 & 9.6 butyl & 96500 & 42700 \\
\hline B & $\mathrm{ZN}^{\mathrm{c}}$ & 126 & 122 & 9.7 butyl & 121000 & 27300 \\
\hline $\mathrm{C}$ & $\mathrm{ZN}^{\mathrm{c}}$ & 126 & 122 & 15 butyl & 140000 & 30000 \\
\hline $\mathrm{Z}$ & $M^{d}$ & 95 & 91 & 28 ethyl & 78600 & 40500 \\
\hline
\end{tabular}

a Peak melting temperature following quenching into ice/water. ${ }^{\mathrm{b}}$ Crystallization temperature; all crystallization times were $30 \mathrm{~min}$. ${ }^{\mathrm{c}}$ Ziegler-Natta. ${ }^{\mathrm{d}}$ Metallocene.

It is of particular interest to examine the structure of rows formed in linear polyethylenes to see what light they can shed on the absence of cellulation in these materials. The first of the three materials listed in Table I to be illustrated is Sclair 2907 chosen as a linear polymer of very similar molecular weight to the LLO209 copolymer but without its high branch content. The essentially uniform growth of the row shown in Figure 6 confirms that it is the presence of branches which induces morphological instability. Growth proceeds in a very regular fashion with lamellae growing radially and more or less parallel right out to the growth tips, clearly a very different growth behavior to the linear-low-density polyethylenes so far considered with no evidence of morphological instability. Notice also the banding beyond the row. This is created on quenching but its linearity, parallel to the nucleating fiber, confirms that the growth interface has remained stable, without protrusions. Barely visible, especially towards the edge of the row, are small pockets of subsidiary lamellae located between, and growing at a substantial angle to, the main lamellae.

This feature recurs in rows of the other two linear polymers. It is most prevalent in Rigidex 9, a material 


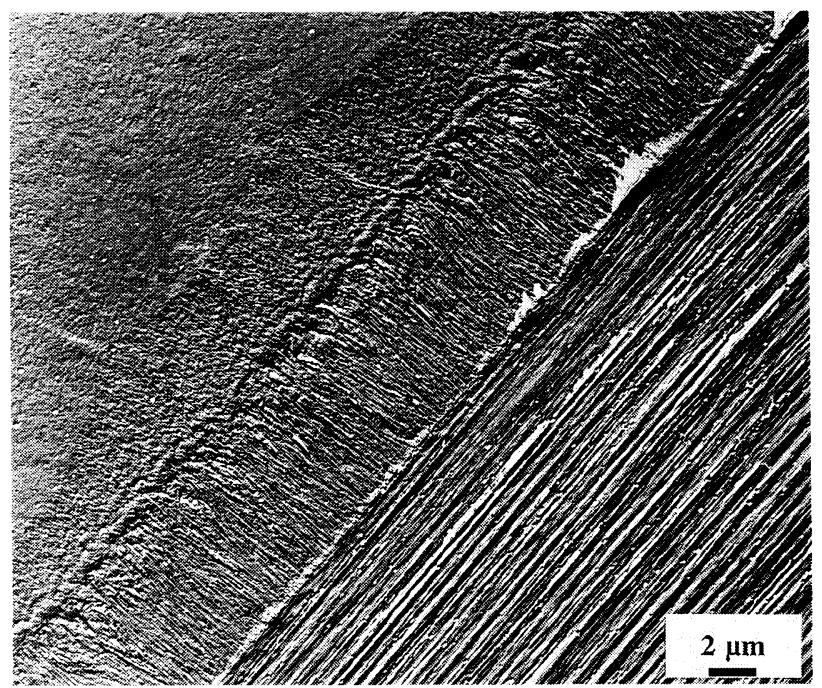

Figure 6. Uniform growth profile of a row in Sclair 2907 showing little or no segregation and a uniform interface demarcated by linear banding produced on quenching.

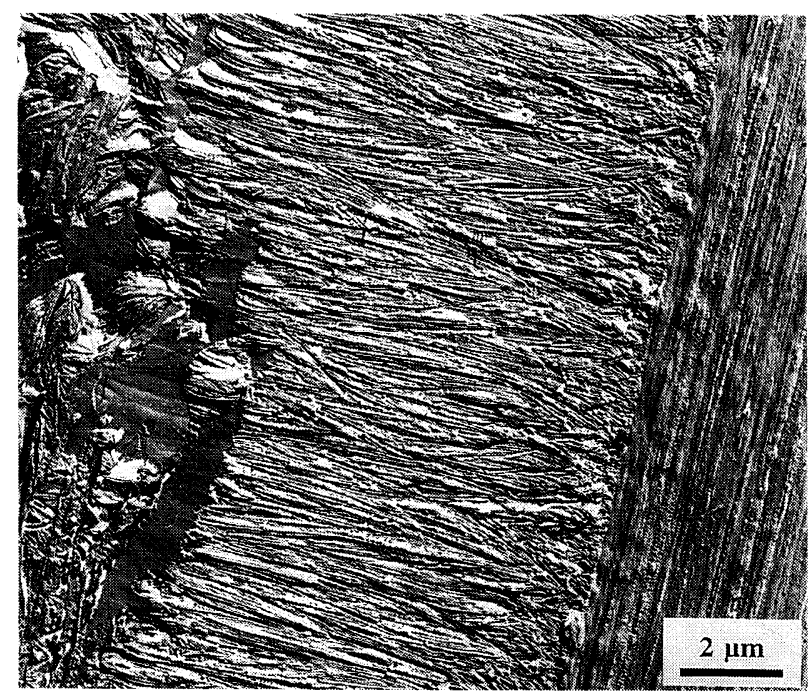

Figure 7. Details of lamellae in a row of Rigidex 140/60 showing predominantly radial lamellae enclosing the occasional pocket of (transverse) subsidiary lamellae.

chosen for its unusually high polydispersity and high molecular weight and marginally less so for the medium molecular weight Rigidex 140/60 which has comparatively narrow polydispersity (Figure 7 ). Note that in this figure the inclination to the radius of lamellae close to the fiber is a consequence of the plane of the specimen not being precisely diametral to the fiber. In consequence the growth direction of lamellae begins to emerge from the page as the fiber is approached so that the viewing direction rotates from being down the $a$ axis to a direction between the $a$ and $b$ crystallographic axes. In this orientation lamellae reveal something of the $\sim 35^{\circ}$ chain inclination characteristic of free growth at low supercooling.

The implication of the small pockets of subsidiary lamellae lying between radial dominants is that, at best, only a minute proportion of these commercial linear polyethylenes is unable to crystallize at $127^{\circ} \mathrm{C}$, a comparatively high temperature. If it were otherwise the re- jected polymer would tend to lie interdigitated between radial dominant lamellae whose separation had increased. The presence of only isolated pockets in these locations-which may or may not contain rejected shorter molecules-rather than a continuous interdigitation puts a very low limit on the extent of segregation which occurs in typical linear polyethylenes. Even were there substantial segregation of shorter molecules, they would have little effect on the local equilibrium melting temperature and, therefore, the growth rate so that cellulation would not be expected. ${ }^{3}$ In any event, as these observations show very clearly, cellulation does not occur in linear polyethylene.

\section{CONCLUSIONS}

1. The segregation of molecules or molecular portions in which branches are too close together to allow crystallization causes a characteristic sequence of morphological changes during the growth of linear-lowdensity polyethylenes (containing in excess of $\sim 10$ branches per $1000 \mathrm{C}$ atoms) from linear nuclei as expected for a progressive decrease in isothermal supercooling. These are coarsening in which lamellae move farther apart on average, allowing growth to deviate from the radial direction together with a degree of thickening, all leading to a corrugated growth interface.

2. The difference in interface geometry between the close-packed lamellar arrays initially present in rows and the more open construction of spherulites reduces the concentration of segregants in the latter and slows the growth less. Spherulites, consequently, grow faster than rows under cellulating conditions in the same sample although the rates are depressed below their initial values in both instances.

3. Ziegler-Natta polymers show an additional feature which has not been observed in metallocene materials namely the nucleation of faster-growing spherulitic protrusions on the instantaneous growth interface. Their presence requires a critical concentration of segregants to be exceeded.

4. The difference between the growth rates of spherulites and rows is distinctly less for metallocene materials than for Ziegler-Natta polymers suggesting that, in the former the segregants are less mobile.

5. Linear polyethylenes show no effects of segregation, cellulation or morphological instability save for the presence of a tiny proportion of small pockets of subsidiary lamellae which may consist of shorter molecules.

6. The first observation of cellulation within spherulites of an undoped polymer has been observed for a metallocene-catalyzed polymer with 28 ethyl branches per $1000 \mathrm{C}$ atoms.

Acknowledgments. The authors are greatly indebted to Dr P Jääskeläinen of Borealis Polymers Oy, Finland for the provision of linear-low-density polyethylenes and to Prof. Keisuke Kaji of the University of Kyoto for Tekmilon fibers. This work formed part of the programme 'Fundamentals of Polymer Crystallization' funded by NEDO. 


\section{REFERENCES}

1. J. J. Janimak and D. C. Bassett, Polymer, 40, 459 (1999).

2. H. D. Keith and F.J. Padden, J. Appl. Phys., 34, 2409 (1963).

3. M. I. Abo el Maaty, I. L. Hosier, and D. C. Bassett, Macromolecules, 31, 153 (1998).

4. M. I. Abo el Maaty, D. C. Bassett, R. H. Olley, and P. Jääskeläinen, Macromolecules, 33, 7800 (1998).

5. J. Varga and J. Karger-Kocsis, J. Polym. Sci., B, Polym. Phys. Ed., 34, 657 (1996).
6. D. R. Fitchmun and S. Newman, J. Polvm. Sci., A-2, 8, 1543 (1970).

7. H. M. White and D. C. Bassett, Polymer, 38, 5515 (1997).

8. M. I. Abo el Maaty, Polym. J., 31, 778 (1999).

9. J. A. Parker, D. C. Bassett, P. Jääskeläinen, and R. H. Olley, Polymer, 35, 4140 (1994).

10. S. C. Mathur and W. L. Mattice, Macromolecules, 20, 2165 (1987).

11. V. B. F. Mathot and T. F. J. Pijpers, Polymer, 39, 979 (1990).

12. M. I. Abo el Maaty to be published. Paper presented at the Polymer Physics Biennial Conference, Bristol (1998). 